\title{
Digital Marketing in the Business Environment
}

Preliminary Communication

\author{
Marin Ištvanić \\ Josip Juraj Strossmayer University of Osijek, \\ Faculty of Electrical Engineering, Computer Science and Information Technology Osijek \\ Kneza Trpimira 2b, 31000 Osijek, Croatia \\ marac1710@mail.hr
}

\section{Dominika Crnjac Milić}

Josip Juraj Strossmayer University of Osijek, Faculty of Electrical Engineering, Computer Science and Information Technology Osijek Kneza Trpimira 2b, 31000 Osijek, Croatia

dominika.crnjac@ferit.hr

\section{Zdravko Krpić}

Josip Juraj Strossmayer University of Osijek, Faculty of Electrical Engineering, Computer Science and Information Technology Osijek Kneza Trpimira 2b, 31000 Osijek, Croatia

zdravko.krpic@ferit.hr

\begin{abstract}
Promotion of products has become an increasingly important component in the new digital age, mostly thanks to digital marketing. The traditional form of marketing is lagging behind digital marketing, which offers users new opportunities like personalized messages or answers to a search query. There are several ways to advertise on the internet, and in this paper, ways and tools will be presented that allow digital advertising as well as their advantages and disadvantages. Specifically, search engine optimization, search engine marketing, display advertising, social networking marketing and e-mail marketing will be discussed. Also, the goal of the paper is to enable more efficient creation and implementation of similar contents in new business environments through an insight into internet advertising, social and business networks.
\end{abstract}

Keywords - display advertising, e-mail marketing, search engine marketing, search engine optimization, social network

\section{INTRODUCTION}

To gain an insight into the growth of the internet over the last fifteen years, it is enough to say that according to [1], by 2016, 3.5 billion people used the internet, while in 2000 that number was only 400 million, which represents a growth rate of $875 \%$. Along with the growth of internet usage, there was a growth of related activities such as digital marketing. The main difference between traditional and digital marketing is that digital marketing focuses on the customer, offering him a personalized message and response to his query.

Many search engines can be used in two equally important ways. The first way is search engine optimization (SEO) that helps companies show unpaid search results. It is the process of optimizing online content so that a search engine shows it as a top result of searches for a certain keyword. Another way is search engine marketing (SEM) that allows marketers to buy inventory in search results. When they are online, besides browsing, users can see various content. In addition to that content, they can also see ads in multiple forms, such as text, image, or video ads. That type of advertising is called display advertising. Additional advertising opportunities on the internet are social networks because they allow connecting with the clients by creating a company profile on one of the social networks where clients are present. In addition to the aforementioned forms of marketing, email marketing can be used, which implies sending information and offers to people who have agreed to receive that kind of emails.

The next section (Section 2) discusses and explains Search engine optimization (SEO) techniques as well as the ones that should not be used. Section 3 deals with the concept of Search engine marketing (SEM) in business by means of an example of a simple Google AdWords auction. Furthermore, Section 4 introduces display advertising and its possibilities on different devices and via different forms of ads as part of digital marketing. Section 5 shows how different social media affect digital marketing methods, which is then accompanied by email marketing evaluated in Section 6. Sec- 
tion 7 discusses digital marketing techniques described in this paper, while Section 8 concludes the paper.

\subsection{RELATED WORK}

As mentioned by Chan, Wu, and Xie (2011) in [12], customers acquired through paid searches purchase more and generate higher customer lifetime value than customers acquired from other online or offline channels. Another topic of this paper was discussed in the paper written in 2015 by Hoban and Bucklin [13], i.e. how the impact of display ads relates to different stages of the purchase funnel. They found a positive impact of display ad exposure on subsequent visits to the company's website. In her paper of 2013 [14], Paquette discussed the idea of using social media as a marketing tool, which is then explained in greater detail in the next paper. The role of email marketing was analyzed by Ellis-Chadwick and Doherty in [15] (2010).

\section{SEARCH ENGINE OPTIMIZATION}

According to [2], search engine optimization is a marketing discipline focused on growing visibility in organic (non-paid) search engine results. Search engines use extremely sophisticated computer programs to classify many websites. By entering a search query into the search engine, a user introduces the search term that the search engine then compares to the catalog of the website. As a result of this process, the search engine on the search results page displays the best search queries from the aforementioned website catalog. As a form of marketing, SEO is very effective because search engines are always available to the user by offering him a solution upon request. The aim of the search engine is to create a list of the most relevant results so that the user can find what he is looking for. As a search result, most of the displayed results are website links, images, maps or videos. These results are mostly displayed at the center of the page and are called either organic or unpaid. Paid results are given above or below these results, and they are tagged as ads and displayed as shown in Figure 1.

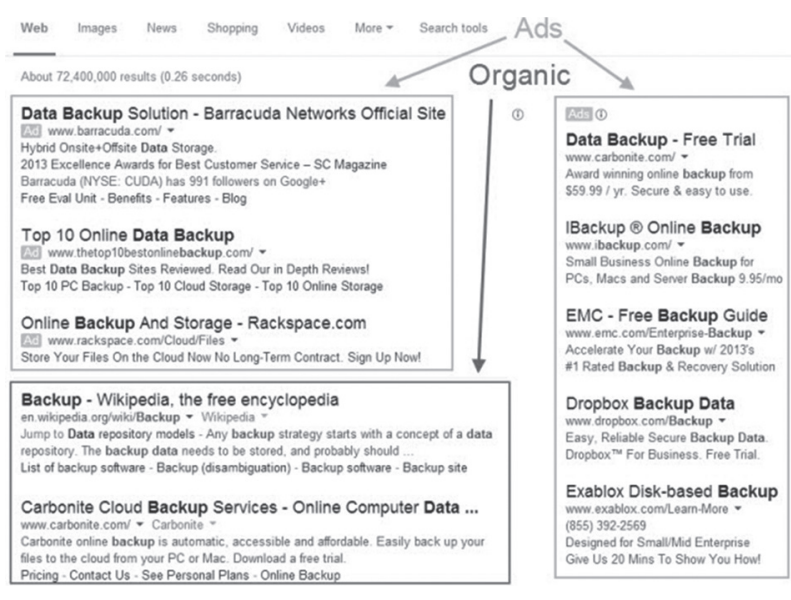

Fig. 1. Organic and paid results

\subsection{SEARCH ENGINE OPTIMIZATION STRATEGIES}

Search engine optimization consists of two strategies, i.e. on-page optimization and off-page optimization. On-page optimization is done for each website separately according to the keywords website visitors enter in the search engine when searching. It includes all actions a company performs on the website to reach a higher rank on the search engine. There are over 200 factors that influence on-page optimization, and according to [3], the most important ones are as follows:

- The number of keywords on the page - Keywords should be used at least three or four times in the page text. The more extensive the page, the more possible it is to repeat keywords;

- HTML title tag < title> - This is one of the most important tags because it tells users and search engines what the subject of a website is. The $<$ title $>$ tag is placed within the $<$ head $>$ HTML document tag and should contain a keyword. It would be ideal to create a unique title for each page of the website;

- Anchor Text - Anchor Text is a text between tags $<\mathrm{a}$ href="..." $></ \mathrm{a}\rangle$. The anchor text for the users and the search engine provides information on the page it is linked to. If the text contains relevant keywords, the search engine considers it an additional and significant recommendation;

- Quality Content - Users like texts that are easy to read and easy to follow, so it is recommended to write high-quality texts. It is also recommended that the person who writes the text is familiar with the topic and uses the relevant but yet not too expert language so that any user can understand it. Keywords should be included in the text, but not too often, because keyword stuffing is the unauthorized practice that violates the terms of business of the search engine.

Off-page optimization follows after on-page optimization and includes all actions done outside of the website. It has a more significant impact on the achieved position in the results after search results than on-page optimization. A backlink plays the most important role among the parameters of off-page optimization.

As noted in [4], backlinks are all links that lead from some other websites to a specific website. It is useful if the original websites are relevant to the landing website. If there are many such links, it is a sign that a lot of different internet users have marked this site as a high-quality one. Search engines will notice this, so the landing website will more often be mentioned in search results.

\subsection{SEO FOR BUSINESS}

SEO is a process itself and it takes time to get the website ranked higher in the search engine results. While SEO is continually changing, it is still an essential 
part of the process of delivering the right content to the right people. SEO helps the audience to find online businesses, and it also guides them through each stage of the buying cycle. It builds awareness because via organic results people can find some website and become aware of the products and services of the business advertised by that website. It also helps people with their research. The more information the website provides through different types of content (like blog posts, infographics and videos), the greater the chance of attracting people to the website. Finally, it helps people to confirm their final choice and entices them into purchasing. The business owner has to build trust and credibility with the customers and convince them to buy from him.

\subsection{BLACK HAT SEO}

Unauthorized practices, popularly called "Black Hat SEO", are a set of techniques used to increase site rankings in search engines by breaking the terms and conditions of the search engine. These are practices that mostly have a short-term effect until they are discovered. Once they have been discovered, their rank is lowered. Some of the examples are:

- Keyword stuffing - too many repetitions of the same keywords to make content more relevant for search engines,

- Link farms - False websites used to inflate the popularity of the original websites using a massive number of fake links to get a better position on search engines.

To prevent Black Hat SEO, Google has developed some algorithms such as "Panda" and "Penguin", which prohibit unauthorized practices by punishing those methods, especially unnatural links, or websites of poor quality.

\section{SEARCH ENGINE MARKETING}

As stated in [4], there are two ways of using search engines for promotion and advertising purposes. The first is SEO, which helps users find the product or company in unpaid search results. An alternative to this is search engine marketing or SEM, where advertisers buy inventory in search results. It is important to note that paid ads do not have any impact on unpaid search results. Unlike traditional marketing, SEM is aimed at people who are actively searching for products or services, so it is vital for advertisers to know which business-related keywords people type while searching. Most search engines use an auction system, in which advertisers bid for keywords and thus compete for ad serving. Advertisers bid on the auction for a limited number of places where ads can appear on the search engine. After the search, the outcome of the auction is presented, as in Table 1, where the user with the highest combination of the bid and quality score gets the highest rank in search results. The bid is the maximum amount the advertiser is willing to pay for the ad click; however, in most cases, he does not pay that amount, but the amount that is sufficient to exceed the competitor participating in the auction. That can be seen in (1), where the ad rank is the rank of the advertiser in the position below and the quality score is a grade of ad quality. In addition to the bid, ad relevance is also crucial. Quality score is a measure rated from 1 to 10 and it shows how closely the ad is related to what the user is looking for. Quality score is an estimate of the quality of an ad and it reflects the relevance of keyword ads, the expected clickthrough rate, and the relevance of the landing page for the ad. As stated in [11], the winner of an auction appears in more desirable places in search results, which means closer to the top. Bidding and creating an ad is done in the search engine user account. An advertising account created in Google AdWords or any other search engine contains campaigns. Each campaign controls essential issues such as a daily budget or search engine networks where ads can be run. Another unique marketing option for search engines is that the advertiser only pays if someone shows interest in the ad, i.e. clicks on the ad. That is why this form of advertising is called pay-per-click (PPC) or cost per click (CPC).

Table 1. Example of an auction in Google AdWords

\begin{tabular}{|c|c|c|c|c|}
\hline & $\begin{array}{l}\text { Max } \\
\text { Bid }\end{array}$ & $\begin{array}{l}\text { Quality } \\
\text { score }\end{array}$ & $\begin{array}{c}\text { Ad } \\
\text { Rank }\end{array}$ & Actual CPC \\
\hline Advertiser 1 & $\$ 2.00$ & 10 & 20 & $16 / 10+\$ 0.01=\$ 1.61$ \\
\hline Advertiser 2 & $\$ 4.00$ & 4 & 16 & $12 / 4+\$ 0.01=\$ 3.01$ \\
\hline Advertiser 3 & $\$ 6.00$ & 2 & 12 & $8 / 2+\$ 0.01=\$ 4.01$ \\
\hline Advertiser 4 & $\$ 8.00$ & 1 & 8 & Highest CPC \\
\hline$C P C$ & $=\frac{}{Q}$ & Adrank & \multicolumn{2}{|c|}{$e+0.01[\$]$} \\
\hline
\end{tabular}

\subsection{GOOGLE SEARCH NETWORK}

The Google Search Network is a group of sites and search-related apps where ads can appear. When advertising on the Google Search Network, ads can appear above or below search results on Google Search, Google Play, Google Shopping, and Google Maps. The text ad in Google Search is the simplest ad type offered by AdWords. It consists of three parts: title, destination URL, and text description, often including ad extensions such as the location or a phone number.

In Google AdWords, an advertiser can choose between manual bidding and automatic bidding. Manual bidding is an option in which the advertiser selects the maximum bid amount for an auction. Automatic bidding is the most commonly used option. By using it, the advertiser defines the daily budget, based on which the AdWords system tries to get most user clicks on the ad within the budget. 
Keywords are words or phrases that match a user's search. When creating a keyword list, it is recommended to look for keywords with high relevance and traffic and with a relatively low cost. Useful tips for optimizing the keyword list are: categorize products into smaller groups, find 5-20 keywords for each category, and use negative keywords.

Shopping ads are more advanced than text ads and are currently available to be shown in search results only for specific countries. They allow users to see a product photo, title or price and therefore provide the customers with a clear idea of the product being sold even before they click on the ad. According to [5], instead of keywords, shopping ads use predefined product attributes.

\section{DISPLAY ADVERTISING}

Unlike search engine marketing, display advertising allows ads to appear on all sites that have inventory. Display advertising provides advertisers with many creative ad ideas - different sizes and formats, images, and videos, which automatically implies more opportunities for attracting potential customers.

\subsection{GOOGLE DISPLAY NETWORK}

According to [4], display advertising is a digital version of jumbo posters or TV ads, and it is used for websites users visit. Companies pay inventory to attract as many potential customers as possible. There are two ways to do that, i.e. by buying ad space from the website owner or by using affiliate networks for companies with different sites promoting ad slots. An ad can be displayed on specific web pages, to an individual website visitor, or both. Like other digital lithographic forms, display advertising platforms offer many options for targeting users. One of the options is to show your ad to the specific speaking area or at a particular time of the day. For example, it is possible to have an exact selection of ad placements by selecting a specific website or specific areas on that website where the ad can appear. Display advertising has many options in defining ad layout. For example, banner ads come in all shapes and sizes, video ads use motion and sound, while Gmail ads are interactive and expandable ads at the top of a user's inbox. An ad banner example is shown in Figure 2 .

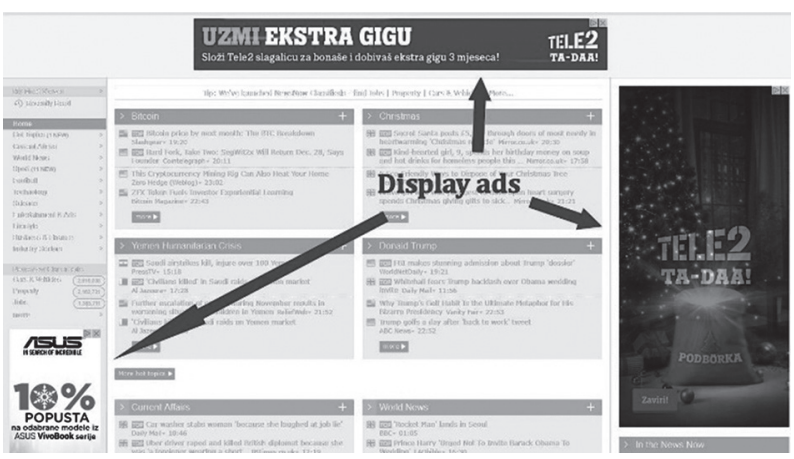

Fig. 2. Example of a display ad

\subsection{CUSTOMER JOURNEY}

Analyzing user steps and imagining this as a funnel can help the advertiser understand what the users want and how to access them. It is possible to divide this stream into the following phases: building awareness, a customer considering the product, a customer reviewing product purchases, and retaining users.

- Building awareness:

- Users do not look for a product actively.

- Broad audience targeting.

- Ads to attract attention of a customer and create an excellent first impression.

- Users considering the product:

- Users have seen the ad.

- Ads that users will remember for the future when there is a need for a purchase.

- Ads that emphasize the advantages over competitors.

- Users consider buying products:

- Users actively interested in the product.

- An additional narrowed audience.

- Ads with a precise message emphasizing the unique advantage of the product.

- User retention:

- Existing customers.

- Ads encouraging users to return to the web page and buy an additional product.

\subsection{REMARKETING}

The most important feature of display advertising is called remarketing, and it allows the use of the user's activity on the advertiser's site to target them with a unique advertising message, even when they leave the site.

There are many remarketing solutions, and many display networks offer this feature. The first step is to define who the target audience is. The target audience can be identified as users who added the product into the cart but did not make any purchase. A remarketing service may require adding a small code to a web page that allows collecting a list of users from the site, i.e., visitors who are suitable for specific criteria and who can later be targeted.

The next step is to create ads tailored to their needs. These ads could include, for example, special offers of additional content, a coupon with a discount, or other incentives to get users back to complete their purchase. When users make their purchase, there is no need to show them the same ad, so a new targeting list for the existing customers should be created. Then they can be targeted with another ad campaign, for example, they can be encouraged to come back and explore other options and products on the site, see [4]. 


\subsection{VIDEO ADVERTISING}

Popularity and an increase in online video quality represent enormous opportunities for advertisers because, according to research from [6], four times more users would rather watch the video of a product than read about it. Videos are quickly becoming the primary marketing tool. The price of an internet connection is much lower, the speed is significantly higher, and there are more ways to reach users on the online video market. Video can be created and shared on a website such as YouTube or advertising space in other people's videos can be bought. Available video ad formats on YouTube include TrueView in-stream ads and TrueView video discovery ads.

TrueView in-stream ads appear before or after videos on YouTube or other sites, in games or apps on the Display Network, and the viewer can skip the ad after five seconds. With TrueView video ad, an advertiser is charged when users watch at least 30 seconds of a video.

TrueView video discovery ads reach people in search results on YouTube or in related YouTube videos. If a viewer clicks on an ad thumbnail, the video will start on YouTube. For this kind of an ad, the advertiser is charged only when a viewer chooses to watch a video, see [5].

\subsection{MOBILE MARKETING}

According to some research [6], today the internet is more accessible through mobile devices than through computers, which is an advantage of mobile marketing and the features it offers. For example, mobile advertising can potentially boost the number of phone calls to the advertiser or encourage people to download mobile apps.

Mobile device users do not search the internet in the same way they search the internet on their computers, so ads should be adjusted to mobile devices. Because mobile phones are smaller, ads should have a concise message with a clear call to action and the ability to appear in the text, picture, or video. In addition to the quality of an ad, it is equally important to have a mobile optimized site because users will more frequently leave a website that is not mobile optimized for users.

The site must work well on smaller screens and load faster because, according to [7], slow loading of a website is the most important reason for abandoning the website. Buttons should be legible and noticeable. Navigation should be clear and user friendly. Visitors should immediately be clear about their options and how to do something. Mobile sites should offer the same things as the main site. An example of a good mobile website is shown in Figure 3 .

In the world of mobile devices, it is not limited to displaying your services solely on websites, but ads can also appear within mobile apps. A mobile app is an application that can be downloaded and installed on a mobile device (cell phone or tablet). It can contribute to a mobile site and help customers achieve specific goals. The application can increase customer loyalty and enable active communication with clients. Applications are also useful for creating loyalty programs and long-term users.
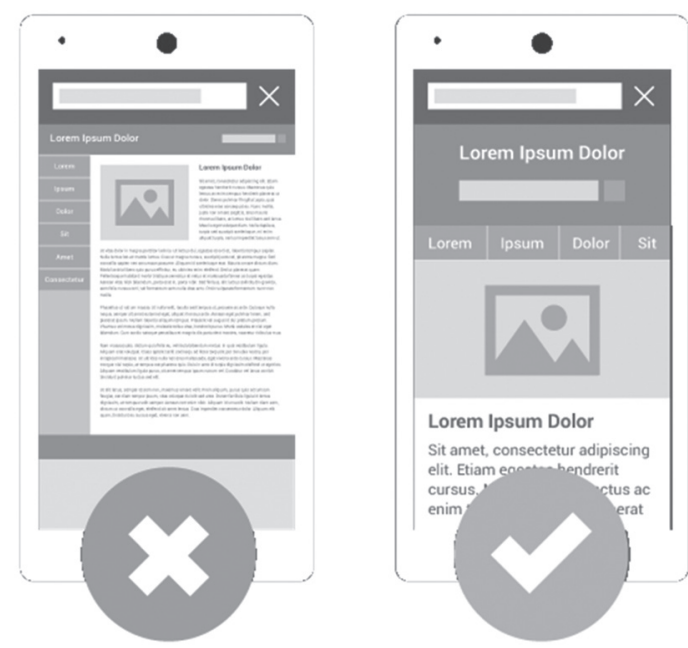

Fig. 3. Example of a bad and a good mobile website

\section{SOCIAL NETWORK MARKETING}

Social networks are present in the lives of internet users on a daily basis, and for companies, they are an excellent opportunity of advertising as they allow users to interact, share and exchange information. Also, social networks can be a great tool for companies as they are platforms that can directly address existing and potential customers, share content, engage in conversations, build trust, reach more people, increase impact, and understand clients better.

Many social networks also offer paid advertising that allows access to specific groups of users and direct advertising to users of these groups because it is more likely for them to become clients than the broader, uninterested audience. Social media sites can do so because they have a lot of information about their users.

The greatest power of social networking is the ability to target potential customers and customers based on demographic information, user behaviors, and specific interests. But besides content promotion, social networking ads are also a great way to increase website traffic or data collection in email campaigns.

\subsection{FACEBOOK}

According to [8], Facebook is the largest social media platform. It has more than 1.9 billion users worldwide. Since Facebook users interact with other users and brands, the platform contains a lot of information about its users. Facebook is one of the most popular network channels for advertisers, because it enables them to utilize rich data about its users to target specific audiences. 
Like AdWords Search campaigns, Facebook ad campaigns are organized on three levels, i.e. ad, ad set and campaign. Creating an ad campaign starts by setting up a campaign goal, then deciding which users to target with the ad, and finally choosing the text, image, and placement of the ad. It is possible to promote unpaid posts to increase one's reach among the existing users or to create a new post as an ad. It is possible to create an ad that has one image, one video, or multiple images within one ad.

Facebook differentiates three different types of objectives that follow a traditional user path from awareness to conversion, through engagement with the post to purchase or install the app. Facebook offers various user-targeting options based on information about their profile:

- Location: target users by country, city, or zip code,

- Demography: target users by gender, age, language and education,

- Interest: target users according to their interests, based on their profile data, joined groups, and pages they interact with,

- Behavior: target users based on their activities and what Facebook knows about their behavior, e.g. how they buy, which mobile devices they own, etc.

When setting a daily budget, the minimum is determined based on what the ad set is charged for. It is possible to pay per click (CPC) or impressions (CPM). The price also depends on the market and the target group advertised. If the target group is very specific, prices are higher than when advertised to a large group of people (see [9]).

\subsection{INSTAGRAM}

Instagram is a mobile social network for sharing photos and videos and, according to [10], it has more than 700 million (monthly active) users in the world. Instagram enables its users to publicly and privately share stock footage and video with Instagram and other social networking platforms such as Facebook or Tumblr. Instagram is a great platform for advertisers because it enables them to tell the company's story visually and excitingly. Successful campaigns at Instagram do not sell products or promote discounts, but they try to tell the story of the product while providing relevant information thereon. Since Facebook took over Instagram in 2012 , both platforms have the same targeting options.

Ads on Instagram can be classified based on how they look like and what their objectives are. All ads on Instagram are placed in the user feed, both in the app and in the browser. There are three types of ads on Instagram: an ad that contains one photo, a video ad, and multiple photos inserted in one ad.

Instagram offers a variety of different objectives a campaign can be optimized for. Like Facebook, Insta- gram shows the ads to users in the target audience who will presumably take action the advertiser wants them to make. The campaign objectives that can be chosen are as follows: building brand awareness, reaching new users, attracting traffic, promoting existing posts, and conversion (on a website or in an app).

\subsection{TWITTER}

Twitter is a social networking platform founded in 2006. Today it has more than 300 million registered (monthly active) users. Twitter users can post short messages not longer than 140 characters called "tweets". Users can add photos, videos and links to their posts. Twitter offers advertisers different types of ads that can be customized according to different goals of the campaign, ranging from increasing the number of site visits and sales to increasing the number of fans. Additionally, Twitter offers the option to address a specific audience, including demographics, interests and behaviors.

Twitter ads are organized according to campaign goals, i.e. actions the advertiser wants the user to take. Some of the types of campaign objectives are:

- Post engagement - A new post or an existing one is promoted to the target audience.

- Grow your followers - This ad type is typical of advertisers who want to promote their Twitter account and increase their follower base. Twitter suggests the target audience to track the advertiser's account and shows them whether one of their followers already follows that account.

- Lead generation - The aim of this type of campaign is to collect user email addresses so that newsletters can be sent to them. It is triggered when a user clicks on a call-to-action button. After that, Twitter submits a name and email address associated with the user's account and displays a custom message. Payment is made for the number of users who clicked on the post.

Twitter requires setting the maximum daily campaign budget, and after this budget is spent, Twitter stops distributing ads. The cost of an ad depends on how many other advertisers have targeted the same audience. Unlike Facebook and Instagram, Twitter does not require setting a minimum budget.

\subsection{PINTEREST}

Pinterest is a social network for sharing photos and videos. It allows its users to upload, sort, organize and save their own pins into two types of collection: collaborative or personal, i.e. virtual boards. According to [8], the platform has about 150 million active users per month. Many companies, especially those associated with interior design, art and fashion, effectively use Pinterest to promote their services and products organically.

Regarding paid advertising, promoted pins are the advertising setup for Pinterest. Promoted pins behave 
and look the same as the regular ones, but advertisers are enabled to pay in order for them to be seen by more users.

Promoted pins can be optimized for three distinct objectives of the campaign, i.e. awareness, engagement, and traffic.

- Awareness campaign - An awareness campaign is a great choice for advertisers whose primary goal is to expose a brand or a product to as many people in the targeted audience as possible. These campaigns are optimized for reach, rather than engagement. Payments are made based on the number of pin impressions.

- Engagement campaign - By choosing this campaign, Pinterest optimizes the distribution of pins, so they are reached by people who might be interested in saving them. Engagement campaigns target people who actively seek solutions to their problems or project ideas. The payment is made for engagement on the pins, not for the views.

- Traffic campaign - This campaign is intended to attract Pinterest users to the advertiser's landing page. This makes it ideal for targeting people who have completed their planning phase and are ready to purchase. This campaign type is the only one with a link to the landing page in the promoted pins. The payment is made for each click on the link.

\subsection{SNAPCHAT}

Snapchat began as a visual messaging service which evolved into a platform that is a combination of the content platform and multimedia messaging. Snapchat currently has more than 150 million monthly active users who share photos and short videos that disappear after the recipient watches them. Advertisers on Snapchat can reach its users via different sponsorships and ad placements. Some of them are:

- $\quad$ Sponsored Geofilters - The majority of Snapchat advertising options focus on large businesses and brands and cannot be booked via platforms like other options that have been mentioned. The only exception is the sponsored Geofilter. These are overlays that have been previously designed (such as images, logos, frames or text elements) that can be used by Snapchat users to decorate images if they reside in a particular location. The price of Geofliters depends on their area size and dates.

- Snap ads - 10-second mobile video advertisements with the ability to discover more associated content, such as ad, mobile website or another video. The Snapchat company states that its users interact with Snap ads five times more than with a comparable platform ad. Snap ad costs depend on campaign details. Snap ads can be booked, but only via Snapchat partners.
- Sponsored Lenses - An intensively interactive format which engages users into advertising elements that overlay users while they are being recorded. Snapchat reports that a typical user interacts for around 20 seconds with a sponsored lens, which contributes to creating product or brand awareness. Due to a high level of user engagement, the price of these effects is quite high, so the sponsored lens can cost $\$ 500,000$ per day.

\subsection{LINKEDIN}

Linkedln is the largest professional social networking platform in the world. It counts over 460 million registered accounts. The primary function of Linkedln is to connect users and search for business contacts. From an advertising point of view, Linkedln is an excellent platform for promoting employers, their jobs and stories, and advertising products and services of interest to the professional audience. It targets users based on their professional experience, education, demographic data and group membership.

Linkedln provides two basic ad types, i.e. Text Ads and Sponsored Content. In addition to these types, larger advertisers can send promotional messages and display ads with customized emails called Sponsored InMail, which they send to targeted users.

The minimum daily budget for both types of ads is $\$ 10$. Linkedln will show the recommended bid range based on other advertiser bids aimed at the same audience. The minimum CPM or CPC bid for the text ad is $\$ 2$, while for sponsored content it depends on the target audience.

\subsection{SOCIAL MEDIA MARKETING FOR BUSINESS}

Marketing via social media is a powerful tool for businesses of all sizes to reach prospects and customers. Customers already interact with various brands by using social media, and it is essential to speak to those prospects through social media platforms like Facebook, Twitter, Instagram and Pinterest directly.

Great social media marketing can bring remarkable success to businesses, creating devoted brand advocates and even driving leads and sales. The bigger and more engaged audience using social media networks, the easier it will be to achieve every other marketing goal. About $90 \%$ of marketers claimed that social media generated extensive exposure of their company, and that is only one of its numerous advantages. Social networks are now a substantial part of every marketing strategy, and the benefits of using social media are so great that anyone not implementing this cost-effective resource is missing a phenomenal marketing opportunity.

The term Social media itself stands for a range of websites, online platforms, internet technologies and digital tools that, at the very least, enable people to: 
- connect with others and participate in online communities around shared interests on the internet,

- share information, content, or opinions with others publicly or closed groups,

- $\quad$ engage in conversations and participate online.

\section{EMAIL MARKETING}

Email marketing is an excellent addition to other activities in digital marketing because without much cost it can build engagement and customer loyalty. According to [4], as in any different type of marketing, the first step in email marketing is to set the goals and create a list of users who have already agreed to receive commercial emails.

Most email marketing tools contain a contact database that must contain at least the email address of a single contact. Furthermore, users should be given the option to cancel their subscription. Online registration forms should be short and simple to complete because this guarantees success. Given the fact that clients receive many emails every day, even the most intriguing message does not guarantee to reach the users. For this reason, it is recommended that content is concise, the sections should not be longer than 5 sentences, and for longer articles and additional information on the website, a call-to-action link should be set up. The text should be more appealing, and the audience should be addressed with the appropriate tone.

Email marketing, as well as other kinds of online marketing, is easy to measure. Reports may contain information such as the email opening rate or the content that attracts most people to the website based on the clickthrough rate. Email campaigns can be improved continuously by testing different versions, creating a relevant landing page and tracking experience analysis.

\section{DISCUSSION}

Out of all techniques described in the paper, SEO is the most important technique for acquiring a customer organically (i.e. free of charge). By optimizing a company website with high quality content, that site can be shown among top places on Google search results. But, since creating content and optimizing it for search engines is a long-term process, lots of companies decide to use a faster way of generating traffic to its websites. Knowing which keyword is often searched by users in combination with a well-created ad, SEM is one of the essential marketing techniques. SEO and SEM are effective because they are aimed at users already showing interest in some of the advertised goods by searching the web. On the other hand, display marketing, even though it provides ads in different sizes and format is not that effective. People are often irritated by display ads, but these ads are still a great way of building awareness for a certain brand. Although ads on social media display ads similarly, they tend to have more success in turning an ordinary reader into a customer. That is because on social media an advertiser can select which group of people to target with his ad based on users' interests and their demographic data. Many digital marketers largely neglect email marketing, but if used adequately, email marketing can have a tremendous effect on users because users are already familiar with the company and its business since they have submitted their email address.

\section{CONCLUSION}

As there exist many ways for product or service promotion, it is rather challenging to choose which one to use. In addition to traditional advertising methods such as television and radio advertising, digital advertising has been on the rise in recent years.

This paper presents digital advertising technologies, techniques and media and their advantages and disadvantages. Understanding the way search engines work and advertising thereon are the most critical items for quality product promotion. For a potential buyer to find a website, it is necessary to optimize it, and thus display the product advertised at the top of search. An alternative to search engine optimization is paid advertising on search engines whose most significant advantage is paying only when a user shows interest in the ad, i.e. clicks on it. Since unlike search engine marketing, users are shown different ads and not just those they are interested in, display ads performance is somewhat weaker, but display advertising is still a great way to create product awareness.

Social networks are also one of the most prominent media advertising channels. They allow everyday interaction with customers and help build awareness of the brand being advertised. The biggest advantage of advertising on social networks are various customer information they have, which makes it easy to find ideal customers. Though the seemingly old advertising model, email marketing allows us to supplement all forms of digital marketing mentioned above, primarily thanks to the possession of email addresses of already interested users and remarketing.

Remarketing is undoubtedly one of the most significant advantages of digital marketing, as it provides the ability to segment the user based on which part of the buying process they are located in and access them with a custom message or an ad for that part of the process. So, it is crucial to know the audience and their habits. Also, unlike traditional marketing, digital marketing is measurable. It is possible to see which ad was interesting to the audience and which failed, and then adjust the advertising strategy accordingly.

Knowing your costs and calculating the amount invested and gained is of great importance to every businessperson, and digital marketing makes that possible. With the development of modern technologies and devices that allow everyday use of the internet, even 
the most traditional entrepreneurs will not resist this form of advertising because it is not only the future but also the present.

\section{REFERENCES:}

[1] Internet Live Stats, Internet Users, www.internetlivestats.com/internet-users, (accessed: 2017)

[2] Seo Administrator, SEO Tutorial, seo-tutorial.seoadministrator.com, (accessed: 2017)

[3] Google, Google's Search Engine Optimization Starter Guide, static.googleusercontent.com/ media/www.google.com/en//webmasters/docs/ search-engine-optimization-starter-guide.pdf (accessed: 2017)

[4] Google Digital Garage, How search engines work, learndigital.withgoogle.com/digitalgarage/lesson/41\#, (accessed: 2017)

[5] Google AdWords, About Search Network only campaigns, support.google.com/adwords/answer/6340430, (accessed: 2017)

[6] D. Chaffey, Mobile Marketing Statistics compilation, www.smartinsights.com/mobile-marketing/ mobile-marketing-analytics/mobile-marketingstatistics, (accessed: 2017)

[7] D. An, P. Meenan, Why Marketers Should Care About Mobile Page Speed, www.thinkwithgoogle. com/marketing-resources/experience-design/ mobile-page-speed-load-time, (accessed: 2017)

[8] Statista, Number of monthly active Facebook users worldwide as of 3rd quarter 2017 (in millions), www.statista.com/statistics/264810/number-ofmonthly-active-facebook-users-worldwide, (accessed: 2017)

[9] Udacity, Social media advertising guide, www. thedigitalrevolutions.in/freedownloads/free-social-media-guide.pdf, (accessed: 2017)

[10] Hub Spot, The Ultimate List of Marketing Statistics, www.hubspot.com/marketing-statistics, (accessed: 2017)

[11] M. Ištvanić, "Analysis and application of digital marketing in the business environment", Josip Juraj Strossmayer University of Osijek, Faculty of Electrical Engineering, Computer Science and Information Technology Osijek, Master Thesis, 2017 (in Croatian)

[12] T.Y. Chan, C. Wu, Y. Xie, "Measuring the lifetime value of customers acquired from Google search advertising", Marketing Science, Vol. 30, No. 5, 2011, pp. $837-850$.

[13] P. R. Hoban, R. E. Bucklin, "Effects of internet display advertising in the purchase funnel: Modelbased insights from a randomized field experiment", Journal of Marketing Research, Vol. 52, No. 3, 2015, pp. 375-393.

[14] H. Paquette, "Social Media as a Marketing Tool: A Literature Review", Major Papers by Master of Science Students, 2013, paper 2.

[15] F. Ellis-Chadwick, F. Doherty, "Web advertising: The role of e-mail marketing", Journal of Business Research, Vol. 65, No. 6, 2010, pp. 843-848. 